\title{
PREVALENCE OF OBESITY AND PHYSICAL ACTIVITY AMONG PRIMARY SCHOOL CHILDREN IN ERBIL CITY/IRAQ
}

Article information

Article history:

Received July 26, 2020

Accepted July 28, 2020

Available online November 8, 2020

DOI: 10.33899/min.2020.164353, College of Nursing, University of Mosul. Creative Commons Attribution 4.0 International License (https://mjn.mosuljournals.com/article_164353.html)

\section{Saad Jbraeil Sulaiman ${ }^{1}$}

Mouroge Hashim AlAni ${ }^{2}$

\begin{abstract}
Background: As inactive lifestyles and a tendency for having a more comfortable life rise in the Iraqi population, obesity prevalence has become a major public health concern particularly among children in the Kurdistan Region Iraq. Moreover, obesity can result in an enormous burden to the economy and public health. Conducting educational programs of physical activity and modifying nutrition patterns among primary school children can be a preventive strategy. The present study was aimed at identifying the obesity prevalence rate and its risk factors among primary school children in Erbil, the Kurdistan Region of Iraq.
\end{abstract}

Patients and methods: In a cross-sectional descriptive study, 400 children who were 6 to 13 years old were selected from primary schools in Erbil and studied from 2017 to 2019. A researcher-designed questionnaire was employed to gather required socioeconomic and sociodemographic data. For this purpose, face-to-face interviews were carried out with the children's parents. Moreover, the UNICEF calibrated digital scale was used to measure the children's weight, a portable stadiometer for their height, and their height and weight for their body mass index. Statistical Package for the Social Sciences (version 22) was used to analyze the collected data.

Results: It was seen that $26 \%$ of the students were obese. All age groups and grades had an almost equal prevalence of obesity. Obesity was more prevalent among those students who were males, had child ranking of $1^{\text {st }}$ or $2^{\text {nd }}$, whose parents lived together, whose parental education was illiterate or primary, whose mother was retired, and whose father was retired. Having an obese mother or father, eating more than 3 main meals per day, physical inactivity, and low socioeconomic status was found to be effective factors in causing children's obesity. There was a significant relationship between children's daily physical activity and nutritional status $(\mathrm{p}=0.000)$.

\footnotetext{
${ }^{1}$ Assistant Lecturer at College of Nursing, University of Duhok.

${ }^{2}$ Pediatric Hematologist Oncologist at Nanakally Hospital \& Professor of Pediatrics at College of Medicine, Hawler Medical Universit
} 
Conclusion: Obesity prevalence was higher among inactive primary school children who had an obese mother or father, ate more than 3 main meals per day and had low socioeconomic status. Daily physical activity of children was directly associated with a decrease in obesity prevalence in children.

Keywords: childhood obesity, physical activity, primary school students, daily meals

\section{INTRODUCTION}

As supported by enormous evidence, physical activity has remarkable health benefits for children and adolescents(Janssen \& LeBlanc, 2010; Organization \& Health, 2010). However, global statistics indicate that the level of physical activity in younger populations is significantly low, and both developed and developing countries refer to physical inactivity prevalence as a major public health concern(Aubert et al., 2018). There is also data showing that there is an increase in sedentary behavior levels and obesity prevalence(González et al., 2018). Global statistics also show that about $80 \%$ of adolescents aged 13 to 15 years do not meet the recommended guidelines as they spend less than 60 minutes of their daily time doing moderate-vigorous physical activity (MVPA) (Bovet, Chiolero, \& Gedeon, 2017). Similar to other developing countries, the Kurdistan region of Iraq is witnessing the development of major health risk factors in its younger populations. According to the results of a study of childhood obesity prevalence among primary school children in Erbil city, obesity and overweight prevalence among the study sample was $1.6 \%$ and $9.3 \%$, respectively [9]. Sherzad also investigated overweight/obesity prevalence and the associated factors, and the results revealed that the overall prevalence of obesity and overweight was 74.3\%(Hallal et al., 2012) .

Moreover, it has been indicated that being overweight/obese is significantly associated with physical inactivity, low educational level, and older age groups of children (Hallal et al., 2012). In addition, as indicated by the results of a largepopulation school-based survey on the physical activity and fitness in China in 2016, the recommended guidelines of 60 minutes of daily MVPA were not met by about $70 \%$ of the school-aged children and adolescents as their average daily MVPA was 45 minutes(Chen, 2017). Another study in China showed that the Chinese national physical fitness "excellent" standards were not met by $94 \%$ of the students, and $12 \%$ were found to be obese according to their body mass index (BMI) percentile scores(Fan \& Cao, 2017). Research has also indicated that boys and girls, adolescents and young children, and children and adolescents living in rural communities and those living in urban communities have several differences with regard to the health outcomes of physical activities (Zhu et al., 2019). As a result, demographic characteristics can be considered as significant associated factors. 
It has been stated that the health goal is to make school-aged children achieve at least 1 hour of physical activity every day and help over $25 \%$ of them achieve an excellent rating in fitness(Fan \& Cao, 2017). However, significant barriers and challenges exist in making progress towards meeting these goals of public health. Some other public health goals are a steady prevalence of physical inactivity in young populations, a constant decrease trend in obesity, an overemphasis on academic excellence, cultural-specific and age-appropriate physical activities, and/or provision of intervention guidelines for school-aged children and adolescents(Fujian, 2018). Such issues have caused the public health surveillance of the Kurdistan Region of Iraq physical activity and other health outcomes to be considered as a high priority, and dealing with these issues is a necessary step towards creating baseline data to inform health promotion strategies and policies . In this regard, the main objective of this study was to provide an update on the population prevalence estimates of physical activity and identifying the obesity prevalence rate and its risk factors among primary school children in Erbil, the Kurdistan Region of Iraq.

\section{METHODS AND MATERIALS}

\section{Study design and setting}

The current study was carried out using a descriptive cross-sectional design. It was aimed at evaluating food behavior and nutritional status among primary school children in Erbil City, the Kurdistan region of Iraq. The study period was from October 1, 2017, to August 1, 2019.

\section{Study sample}

A multi-stage approach, including cluster, stratified, and simple random sampling was employed to select 400 primary school students from among a target population of 6,614 students. In so doing, based on Erbil Municipalities, six geographical sectors in Erbil city were determined. Afterward, a randomized sampling method was used to choose two primary schools in each sector. Finally, using stratified sampling, grades one to six of primary schools were selected, and to pull near 5\% from each school strata, a simple random sampling was employed, which led to the selection of 400 students.

\section{Data collection}

A researcher-designed questionnaire was used to collect the required data. It consisted of two sections. The first section included items regarding the children and their parents' demographic characteristics, while the second section related to weekly child's physical activities which are included with 19 dichotomous questions with yes or no responds and 6 multiplechoice questions. The of the families were measured through another questionnaire which provided data on the parents' 
educational level, number of family members, crowding index, type of house and properties. The socioeconomic status scores of 70-100 were labeled as high, 4069 as moderate, and 10-39 as low. Face-toface interviews were conducted with the children's parents at their home in order to complete the questionnaires.

A UNICEF calibrated digital scale (UNICEF electronic scale Seca 890) was used to measure the children's weight to the nearest 0.1 kilograms while the children were wearing light clothes and no shoes. Also, a portable stadiometer (Seca
208 body meter, secavogel and $\mathrm{GmbH}$, and Co, Hamburg, Germany) was employed to measure their height to the nearest centimeter while the children were asked to stand without wearing any shoes. Moreover, the following equation was used to calculate their body mass index:

BMI=weight $(\mathrm{kg}) /[$ height $(\mathrm{m})] 2$

In addition, an international standardized chart according to gender-specific BMI form CDC 2-20 years were used to determine their BMI. For this purpose, the following table was used to determine their weight status.

Table 1 . Weight status category percentile range

\begin{tabular}{c|c}
\hline Underweight & BMI $<5$ th percentile \\
\hline Healthy weight & BMI for age $\geq 5$ and $<85$ percentile \\
Overweight & BMI for age $\geq 85$ and $<95$ th percentile \\
Obese & BMI $\geq 95$ th percentile
\end{tabular}

To examine the validity of the questionnaire, it was given to 10 experts who had experience in the field, and the final draft of the questionnaire was revised based on their views. Moreover, a pilot study was conducted to check its reliability. For this purpose, a randomly chosen sample of 30 primary school children was used, which revealed its reliability to be $r=0.790$.

\section{Data analysis}

Statistical Package for the Social Sciences (version 22) was used for data analysis. The association between the children's socio-demographic status and nutritional status was also determined. Moreover, the relationship between their nutritional status and food behavior was examined by using regression.

\section{Ethical consideration}

Required approval was obtained from the College of Nursing, Hawler Medical University, the Kurdistan Region of Iraq. In addition, the Directorate of Education in Erbil gave necessary approval. Moreover, each parent announced his/her informed consent.

\section{RESULTS}

According to the results, obesity was observed in all groups with somewhat 
Mosul Journal of Nursing, Vol. 8, No. 1, 2020 ( 1-13 )

close percentages. However, the highest percentage rate of obesity belonged to the age group $12-<13$ years old $(31.66 \%)$. It was also seen that obesity was more prevalent among male students $(n=60)$ that the females $(n=44)$. It was also found that all grades suffered from obesity almost equally, with the highest rate belonging to the 6 th grade with a percentage of $33.3 \%$. Children who were the 1 st or 2 nd child in their families had the highest rate of obesity with $32.3 \%$. The results showed that obesity was more prevalent among children whose father got married more than once, followed by those whose parents were living together. Obesity was more prevalent among children whose mother Illiterate and whose father had a primary level of education. Children whose parents were a retired had the highest prevalence of obesity (See Table 2). 
Mosul Journal of Nursing, Vol. 8, No. 1, 2020 ( 1-13 )

Table 2. Distribution of socio-demographic data of primary school children $(n=400)$

Variables

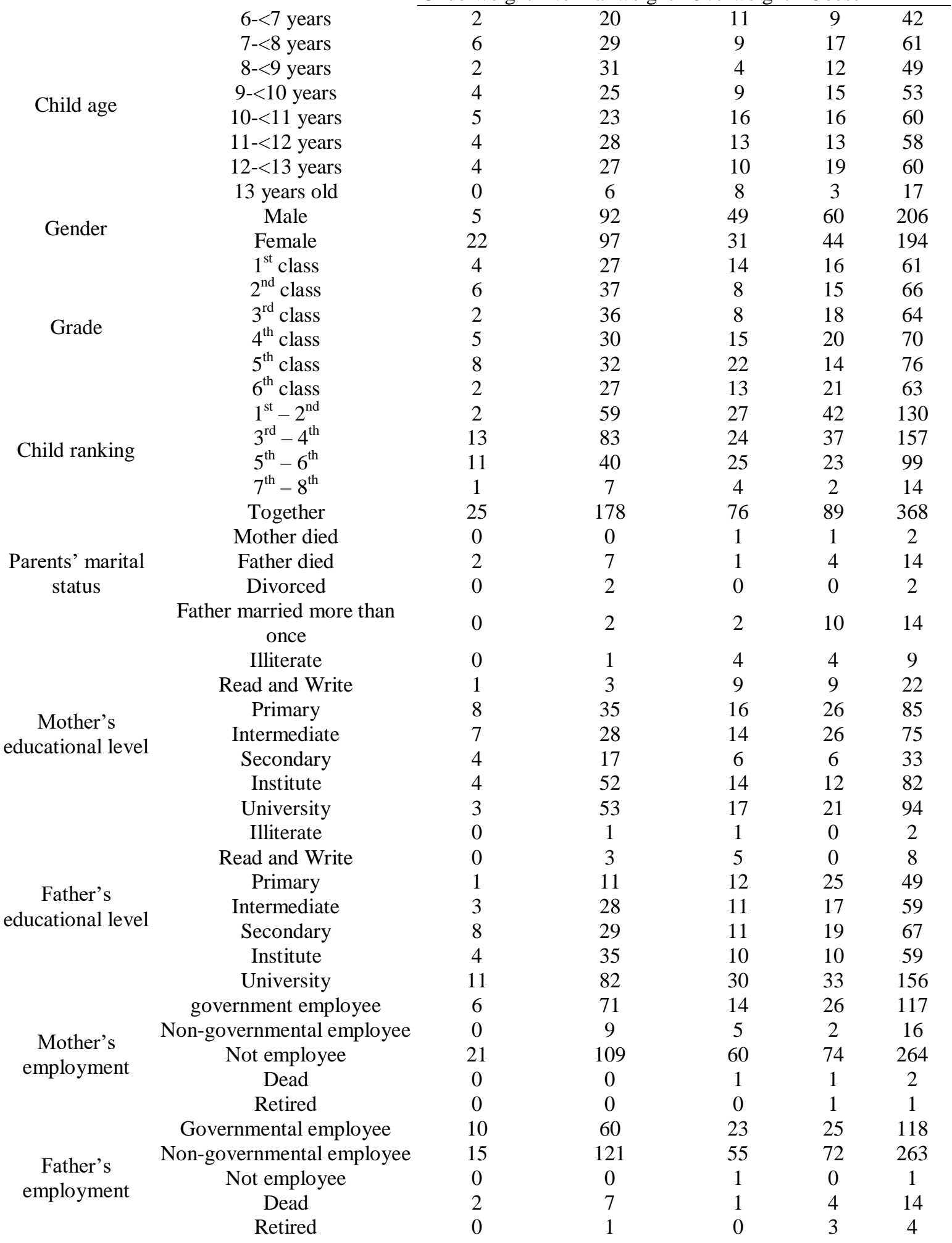

Table 3. Distribution of weight status for primary school students 
Mosul Journal of Nursing, Vol. 8, No. 1, 2020 ( 1-13 )

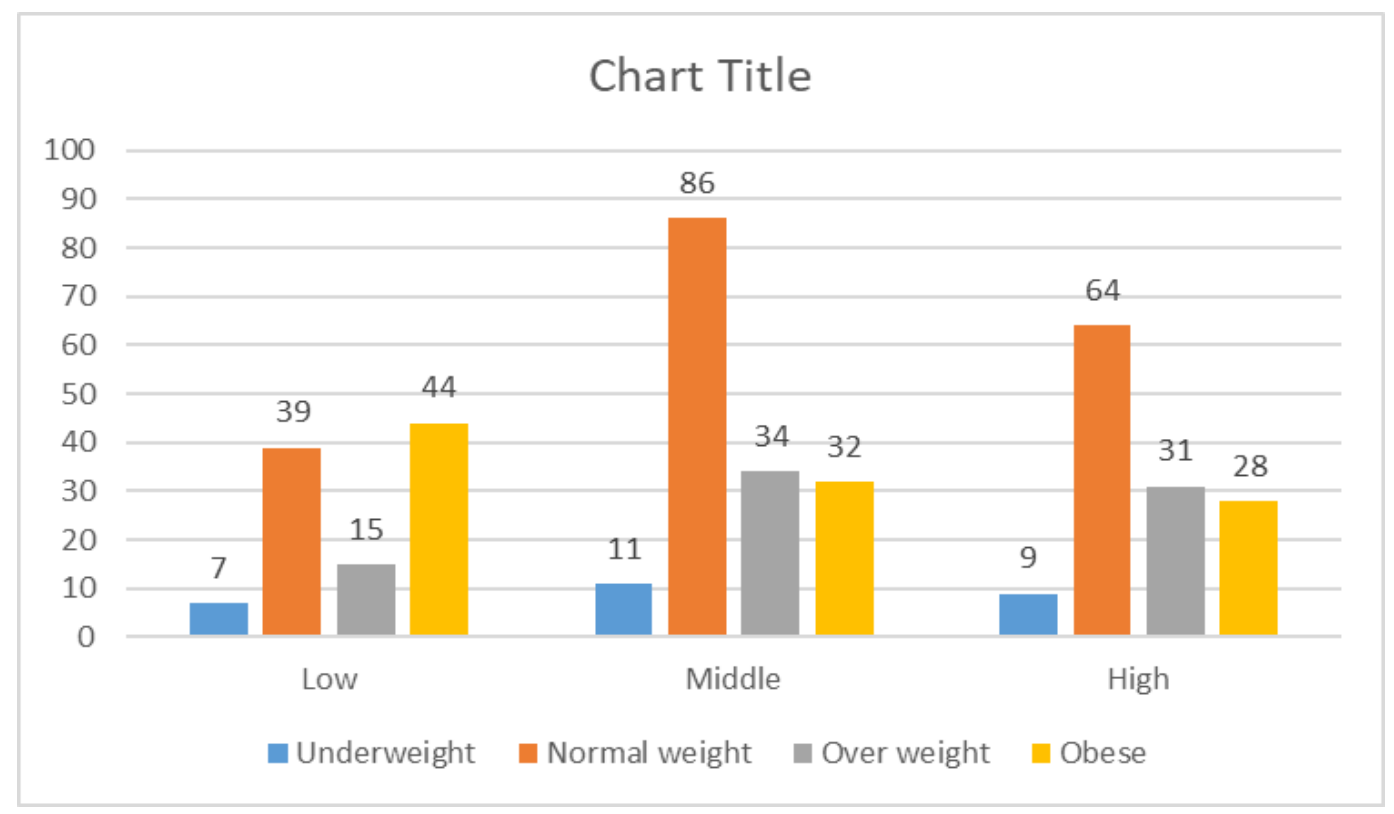

Figure 1. Distribution of weight status of children with their socioeconomic status

The data collected on the students' family history of obesity indicated that the highest prevalence of obesity belonged to those children whose mother or father was obese (See Table 4). The results of the study revealed that there was a highly significant association between the occurrence of obesity and the number of the main meals eaten by the children ( $p$-value $=0.000$ ), such that obesity was more prevalent among those students who ate more than 3 main meals per day, followed by those who ate 3 times a day (See Table 5).It was also observed that obesity was more prevalent among inactive students compared to active ones with 80 students (35\%), while only 24 active students were obese (See Table 6).The results also indicated that there was a highly significant association between the children's daily activity and their nutritional status $\quad(\mathrm{p}$-value $=0.000) \quad($ See Table 7).Moreover, as revealed by the results of the study, the obesity rate was remarkably higher among children whose families had a low socioeconomic status, such that 44 out of 104 students were obese (see Figure 1).

\section{DISCUSSION}

The trends of overweight/obesity incidences in school children have undergone a remarkable rise over time(Pangani, Kiplamai, Kamau, \& Onywera, 2016). As research indicated, obesity can result from several factors, including the adoption of unhealthy 


\section{Mosul Journal of Nursing, Vol. 8, No. 1, 2020 ( 1-13 )}

dietary patterns, inappropriate lifestyle, physical inactivity, biological factors like alcohol consumption, consumption of some medications, age, gender, and race, and some diseases(Khazaei et al., 2017). Based on the results of the present study, it could be seen that the majority of children who participated in this study had normal weight, $20 \%$ of them were overweight, and $26 \%$ of them were obese. As shown by Salman et al, the most important factor among primary school children which may be easily controlled by conducting proper educational courses is a physical activity (Khazaei et al., 2017). In addition, based on the results reported by Ismail et al, overweight/obesity prevalence is higher in females than males(Sulaiman \& AlAni, 2020). As opposed to this result, the results of the present study revealed that overweight/obesity prevalence was higher among males, which might be because of different factors associated with obesity incidence in the two study samples. However, the findings of this study are in line with those of O'Dea in Australia in 2008 (O'Dea, 2008) and that of HajianTilaki in Iran in 2011(Hajian Tilaki, Sojjodi, \& Razavi, 2011) who found that male children had more prevalence in obesity than the females. Another factor that was detected to be effective in the obesity situation of the students was the living situation of children's parents. In this regard, obesity was found to be more prevalent among children whose fathers got married more than once and parents were living together. This finding is in line with those reported by Philip and David who demonstrated that in families where parents live together the rate of obesity among children is higher which may be due to their different and richer diet plans. The parents' education level was another factor that affected the obesity situation of children, such that children whose parents had Illiterate or a primary level of education had a higher rate of obesity. These results are in line with those reported by Sherzad who worked on the prevalence of overweight/obesity and associated factors in Erbil, Iraq and demonstrated that there was a significant statistical association between being overwei-ght/obese and low educational level of the children's parents (Shabu, 2019). Investigating the association of parents' employment status and children's obesity situation in different studies has led to different results. For example, the studies conducted by Salman et al and Philip and David showed that the parents' employment status could significantly impact the obesity situation of children.(Khazaei et al., 2017). In accordance with the data presented in the present study, it could be seen that the rate of obesity among children whose father was retired was higher. Similarly, among children whose mothers were not employees anywhere, the obesity rate was higher in comparison with others. Some other studies demonstrated various results, for instance, the prevalence of obesity 


\section{Mosul Journal of Nursing, Vol. 8, No. 1, 2020 ( 1-13 )}

among children whose parents were employed was higher than those with unemployed parents(Musaiger, 2011). However, the data presented by Sherzad demonstrated that the obesity rate among children whose parents were unemployed was higher than those with employed parents (Shabu, 2019). In accordance with the results reported by Philip and David, family-based interventions for childhood obesity would be the most efficient intervention for obese children whose parents have severe and complicated obesity. In this regard, they reported that the potential of being overweighed/obese among children whose parents were overweight or moderately obese was found to be higher(McLoone \& Morrison, 2014). In line with this, the results obtained in the present study demonstrated that the highest prevalence of obesity belonged to those children whose mother or father was obese. The majority of the participants in the present study had unemployed mothers, which can be a justification for the fact that the consumption of junk food among the school children of the present study was not high. Anyway, the results of the study demonstrated that there was a highly significant association between the occurrence of obesity and the number of the main meals eaten by the children, and the rate of obesity among children who ate three or more main meals per day was more prevalent. In line with the results of the present study, Abdulrahman reported that students who eat more than 3 meals per day were overweight or obese(Musaiger, 2011) . Moreover, Morrissey et al emonstrated that working mothers that can afford junk food for their children may cook fewer meals at home, thus opting for more restaurant meals and fast foods that are densely packed with calories, therefore cause overweight and/or obese among children (Morrissey, Dunifon, \& Kalil, 2011)

Investigations into the effect of physical activities on obesity status should specify certain kinds of influential activities, rather than only examining "light, moderate, or heavy" tendencies in evaluating energy expenditure(Zalilah, Khor, Mirnalini, Norimah, \& Ang, 2006). Also, different methods need to be examined for a precise calculation of the time spent on different activities. One of these methods can be the direct observation or record of physical activities by the parents, rather than merely asking the children (Zhu et al., 2019). The data presented in the present study demonstrated that obesity was more prevalent among inactive students compared to active ones, and the rate of active students who were obese was low. Based on the results of the study carried out by Abdulrahman et al, the obesity prevalence among the students in the Kurdistan Region of Iraq is not high in comparison to Western countries. However, due to the changes in lifestyle over recent years, it is vital to devise physical intervention programs within 
Mosul Journal of Nursing, Vol. 8, No. 1, 2020 ( 1-13 )

schools and families to improve physical activity and dietary patterns of this age group(Musaiger, Al-Mannai, \& Mufty, 2014). Moreover, it should be noted that there is a high association between children's daily activity and their nutritional status. Therefore, the constant use of physical activity and fitness protocols to monitor these behavioral health outcomes is also needed to guide policies and programs aimed at increasing physical activity and reducing obesity among the school-aged children and adolescents in the Kurdistan Region of Iraq. On the other hand, the obesity rate could be remarkably decreased among children as a result of improvement in the families' socioeconomic status(Organization \& Health, 2010; Wang, 2017).

\section{CONCLUSION}

This study investigated the prevalence of obesity and physical activity among primary school children in Erbil City/Iraq and demonstrated that the prevalence of obesity was different based on various age groups and among students who had various physical activity patterns. Due to changes in lifestyle, especially over recent years, it is essential to plan and implement intervention programs to improve the lifestyles and dietary patterns of this age range in their families, schools, and communities. Moreover, early interventions on these modifiable risk factors are likely to reduce the rate of childhood obesity. School health programs by concerned stakeholders in promoting the low intake of junk food, active commuting to school, and active lifestyle among children are recommended. Further studies with objective measurements to identify the association between diet- and activity-related behaviors and childhood overweight/obesity among the school children in the Kurdistan Region of Iraq are highly recom-mended. 


\section{Mosul Journal of Nursing, Vol. 8, No. 1, 2020 ( 1-13 )}

\section{REFERENCES}

Aubert, S., Barnes, J. D., Abdeta, C., Nader, P. A., Adeniyi, A. F., Aguilar-Farias, N., . . . Cardon, G. (2018). Global matrix 3.0 physical activity report card grades for children and youth: results and analysis from 49 countries. Journal of physical activity and health, 15(s2), S251-S273.

Bovet, P., Chiolero, A., \& Gedeon, J. (2017). Health Effects of Overweight and Obesity in 195 Countries. The New England journal of medicine, 377(15), 1495-1496.

Chen, P. (2017). Physical activity, physical fitness, and body mass index in the Chinese child and adolescent populations: an update from the 2016 Physical Activity and Fitness in China-The Youth Study. Journal of sport and health science, 6(4), 381.

Fan, X., \& Cao, Z.-B. (2017). Physical activity among Chinese schoolaged children: national prevalence estimates from the 2016 Physical Activity and Fitness in China-The Youth Study. Journal of sport and health science, 6(4), 388-394.

Fujian, L. (2018). 2030 Vision for ASEANChina Strategic Partnership: Perspectives from Think-Tanks: World Scientific.

González, S. A., Barnes, J. D., Nader, P. A., Tenesaca, D. S. A., BrazoSayavera, J., Galaviz, K. I., . . . Liu, Y. (2018). Report Card Grades on the Physical Activity of Children and Youth From 10 Countries With High Human Development Index: Global Matrix 3.0. Journal of physical activity and health, 15(s2), s284-S297.

Hajian Tilaki, K., Sojjodi, P., \& Razavi, A. (2011). Prevalence of overweight and obesity and associated risk factors in urban primary-school children in Babol, Islamic Republic of Iran. EMHJ-Eastern Mediterranean Health Journal, 17 (2), 109-114, 2011.

Hallal, P. C., Andersen, L. B., Bull, F. C., Guthold, R., Haskell, W., Ekelund, U., \& Group, L. P. A. S. W. (2012). Global physical activity levels: surveillance progress, pitfalls, and prospects. The lancet, 380(9838), 247-257.

Janssen, I., \& LeBlanc, A. G. (2010). Systematic review of the health benefits of physical activity and fitness in school-aged children and youth. International journal of behavioral nutrition and physical activity, 7(1), 40.

Khazaei, S., Mohammadian-Hafshejani, A., Nooraliey, P., Keshvari-Delavar, M., Ghafari, M., Pourmoghaddas, A., . . . Sarrafzadegan, N. (2017). The prevalence of obesity among school-aged children and youth aged 6-18 years in Iran: A systematic review and metaanalysis study. ARYA atherosclerosis, 13(1), 35.

Mcloone, P., \& Morrison, D. S. (2014). Risk of child obesity from parental obesity: analysis of repeat national cross-sectional surveys. The European Journal of Public Health, 24(2), 186-190.

Morrissey, T. W., Dunifon, R. E., \& Kalil, A. (2011). Maternal employment, work schedules, and children's body mass index. Child development, 82(1), 66-81.

Musaiger, A. O. (2011). Overweight and obesity in eastern mediterranean region: prevalence and possible causes. Journal of obesity, 2011.

Musaiger, A. O., Al-Mannai, M., \& Mufty, B. A. (2014). Prevalence of overweight and obesity among adolescents in $E$ rbil, I raq. 
Nutrition \& Dietetics, 3(71), 213214.

O'Dea, J. A. (2008). Gender, ethnicity, culture and social class influences on childhood obesity among Australian schoolchildren: implications for treatment, prevention and community education. Health \& social care in the community, 16(3), 282-290.

Organization, W. H., \& Health, W. H. O. R. (2010). Medical eligibility criteria for contraceptive use: World Health Organization.

Pangani, I. N., Kiplamai, F. K., Kamau, J. W., \& Onywera, V. O. (2016). Prevalence of overweight and obesity among primary school children aged 8-13 Years in Dar es Salaam city, Tanzania. Advances in preventive medicine, 2016.

Shabu, S. A. (2019). Prevalence of overweight/obesity and associated factors in adults in Erbil, Iraq: A household survey. Zanco Journal of Medical Sciences (Zanco J Med Sci), 23(1), 128-134.

Sulaiman, S. J., \& AlAni, M. H. (2020). Prevalence of obesity and physical activity among primary school children in Erbil City/Iraq. Mosul Journal of Nursing, 8(1), 6-17.

Wang, D. (2017). Improving school physical education to increase physical activity and promote healthy growth of Chinese schoolaged children-Time for action. Journal of sport and health science, 6(4), 384.

Zalilah, M. S., Khor, G., Mirnalini, K., Norimah, A., \& Ang, M. (2006). Dietary intake, physical activity and energy expenditure of Malaysian adolescents. Singapore medical journal, 47(6), 491.

Zhu, Z., Tang, Y., Zhuang, J., Liu, Y., Wu, X., Cai, Y., . . . Chen, P. (2019). Physical activity, screen viewing time, and overweight/obesity among Chinese children and adolescents: an update from the
2017 physical activity and fitness in China-the youth study. BMC Public Health, 19(1), 197. 